\title{
Simulations of Ion Trapping in a Micrometer- Sized Cylindrical Ion Trap
}

\author{
Daniel E. Austin \\ Department of Chemistry and Biochemistry, Brigham Young University, Provo, Utah, USA
}

\author{
Dolores Cruz and Matthew G. Blain \\ Microanalytical Systems, Sandia National Laboratories, Albuquerque, New Mexico, USA
}

\begin{abstract}
We have performed detailed SIMION simulations of ion behavior in micrometer-sized cylindrical ion traps $\left(r_{0}=1 \mu \mathrm{m}\right)$. Simulations examined the effects of ion and neutral temperature, the pressure and nature of cooling gas, ion mass, trap voltage and frequency, space-charge, fabrication defects, and other parameters on the ability of micrometer-sized traps to store ions. At this size scale voltage and power limitations constrain trap operation to frequencies about $1 \mathrm{GHz}$ and rf amplitudes of tens of volts. Correspondingly, the pseudopotential well depth of traps is shallow, and thermal energies contribute significantly to ion losses. Trapping efficiency falls off gradually as $\mathrm{q}_{\mathrm{z}}$ approaches 0.908 , possibly complicating mass-selective trapping, ejection, or quantitation. Coulombic repulsion caused by multiple ions in a small-volume results in a trapping limit of a single ion per trap. If multiple ions are produced in a trap, all but one ion are ejected within a few microseconds. The remaining ion tends to have favorable trapping parameters and a lifetime about hundreds of microseconds; however, this lifetime is significantly shorter than it would have been in the absence of space-charge. Typical microfabrication defects affect ion trapping only minimally. We recently reported (IJMS 2004, 236, 91-104) on the construction of a massively parallel array of ion traps with dimensions of $\mathrm{r}_{0}=1 \mu \mathrm{m}$. The relationship of the simulations to the expected performance of the microfabricated array is discussed. (J Am Soc Mass Spectrom 2006, 17, 430-441) () 2006 American Society for Mass Spectrometry
\end{abstract}

I $n$ parallel with advances in micromachining technology, numerous efforts are underway to produce miniaturized and microfabricated analytical instrumentation. Although portable instruments rarely match the performance of larger laboratory instruments, the benefits of low mass and power make small instruments well-suited for real-time in-field analytical applications. Mass spectrometers are attractive targets for miniaturization because they generally exhibit high sensitivity and chemical specificity. Many groups have focused on miniaturizing mass spectrometers, and small mass analyzers based on time-of-flight [1-6], magnetic sector [7], linear quadrupole [8], and hyperbolic and cylindrical ion traps [9-17] have recently been described. The dimensions of some of these mass analyzers are about a millimeter or smaller. As mass analyzer dimensions decrease, vacuum requirements also typically decrease due to the shorter ion path and correspondingly shorter tolerable mean free path [11, 18]. Similarly, power requirements typically decrease as the sizes of capacitive and inductive components are

Published online January 25, 2006

Address reprint requests to Dr. D. E. Austin, Department of Chemistry and Biochemistry, Brigham Young University, Provo, UT 84602, USA. E-mail: austin@chem.byu.edu

reduced, or as the required voltage needed for a given field strength decreases. Further reductions in size, for example, down to the micrometer range, may allow sufficiently small pumping, power, and electronics packages as to make a truly hand-held instrument.

We recently reported [19] on the design considerations and development of microfabricated arrays of cylindrical ion traps for use as mass analyzers. These arrays contain up to $10^{6}$ traps $/ \mathrm{cm}^{2}$. The geometry of the cylindrical ion trap is amenable to multi-level microfabrication techniques, and the trap size and electrical requirements make it suitable for layout as a large array. Figure 1 shows a SEM micrograph of a cylindrical ion trap array produced in our laboratory. Ion traps of this size have several limitations not present in larger ion traps. For instance, the voltage difference between electrodes must remain below the field emission limit [20], but must be large enough that the pseudopotential well depth will trap ions. Current is limited by device wirebonding, and power is limited by the ability of a microfabricated device to dissipate heat. The corresponding frequencies needed to trap ions consequently may be about $\mathrm{GHz}\left(10^{9} \mathrm{~Hz}\right)$ for a trap with $\mathrm{r}_{0}=1 \mu \mathrm{m}$. As ohmic heating of rf electrical circuits is dependent on the capacitive reactance portion of the impedance, capacitive coupling between electrodes-directly as well 
Top View of Trap Array

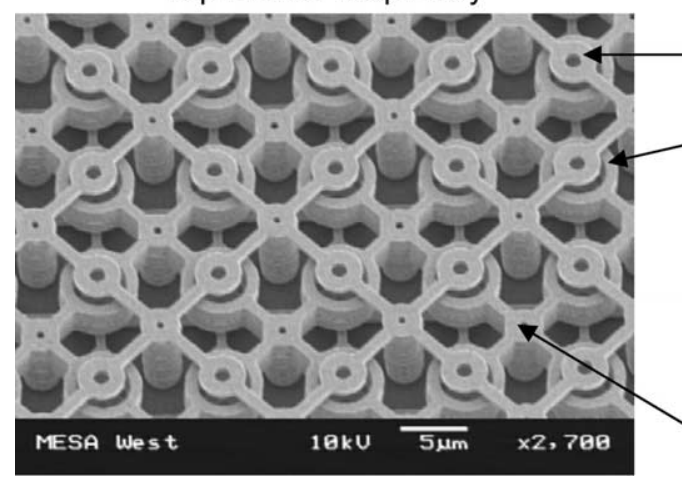

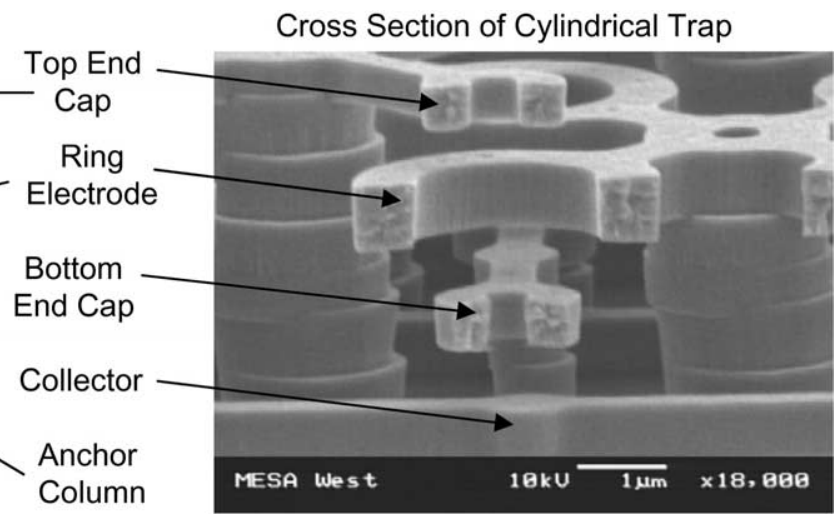

Figure 1. SEM micrographs of sections of a microfabricated cylindrical ion trap array produced in the Microelectronics Development Laboratory at Sandia National Laboratories.

as through the substrate-must be minimized. Fabrication defects such as misalignment between layers, tapering, and surface roughness must also be taken into account. On the other hand, it may be possible to include the mass analyzer, detector, and both ion signal collection and drive electronics on a single chip, resulting in significant space and weight savings. In addition, because the ion motion occupies smaller dimensions, the operating pressure may be higher than in larger mass analyzers [21].

Numerous studies have examined ion trapping behavior in macroscopic traps of various geometries, including cylindrical traps [22-32]. Experimental results are also available for cylindrical traps with dimensions down to $1 \mathrm{~mm}[12,14-16,33-35]$. Preliminary results have been presented for traps with $\mathrm{r}_{0}=40 \mu \mathrm{m}$ [17]. However, ion behavior in the sub-millimeter range has not been studied. To understand the ion trapping limitations resulting from the small trap size we have performed detailed computer simulations, which focus on the effects of temperature, pressure, voltage, frequency, and other parameters on the ability of micrometer-sized traps to store ions. Ion ejection and mass analysis are alluded to here but are not discussed in detail. Preliminary results of some of these simulations were presented in a previous publication [19], to which this paper is a follow-up. Specifically, this paper presents additional data on the effects of temperature, the trapping parameter qz, and pressure of cooling gas, which were presented in the previous work.

\section{Computational Method}

Simulations of ion trap performance were carried out using SIMION 7.0 ion trajectory software [36]. Micrometer-scale ion traps were defined in SIMION using a three-dimensional nonsymmetric 5-millionpoint potential array using the actual etch mask dimensions of our first prototype microfabricated traps, including the mechanical-electrical connections leading up to the trap electrodes. Trap dimensions and an image of the electrode model are shown in Figure 2.
Some simulations used traps with ideal fabrication, while other studies examined the effect of fabrication defects such as electrode sidewall tapering and electrode misalignment. The potential arrays were refined to a convergence limit of $10^{-7}$, the lowest limit possible in SIMION. An extensive user-written code established time-varying electric potentials, initial ion parameters, collisions, and control functions. During the simula-
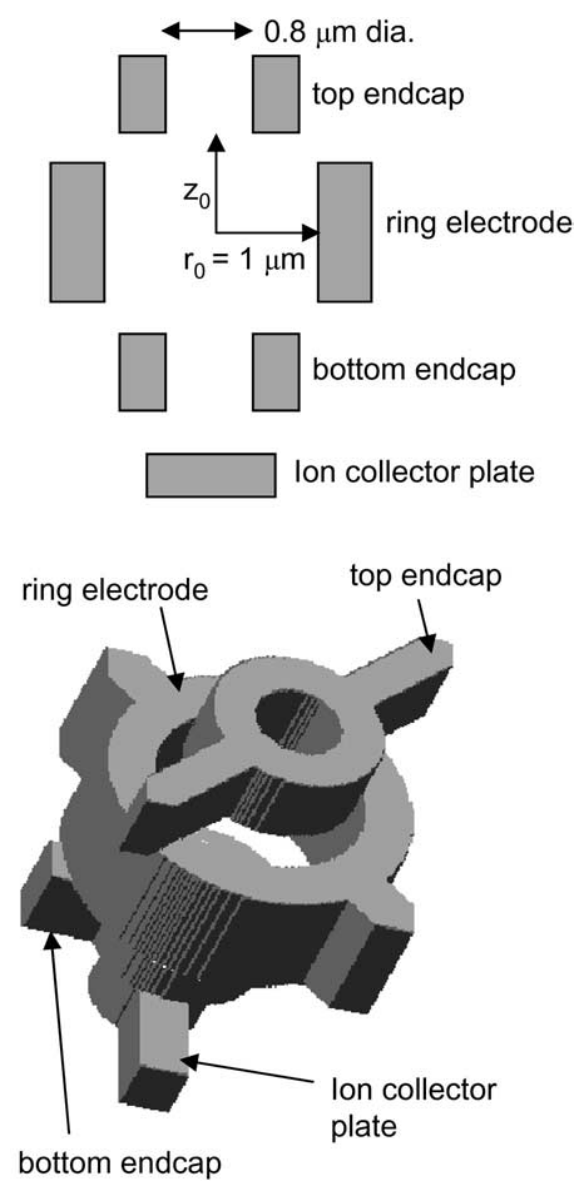

Figure 2. Diagram and image of the cylindrical ion trap used in simulations. 
tions, the potential energy surfaces were updated no less than 50 times each $\mathrm{rf}$ cycle to ensure that the $\mathrm{rf}$ potential was modeled adequately. An overview of this code, including key algorithms, is included in the Appendix. This same code, and identical trap geometry, was also used in reference [19].

Ionization is assumed to take place in situ [19] and the initial positions of ions were randomized (uniformly distributed with respect to $\mathrm{x}, \mathrm{y}$, and $\mathrm{z}$ ) within a cylindrical volume, with radius equal to that of the aperture in the end cap electrode $(0.4 \mu \mathrm{m})$, and with length equal to the distance between the inner surfaces of the end cap electrodes $(0.95 \mu \mathrm{m})$. This corresponds to the volume accessible to electron impact ionization in the specific ion traps we have produced. If the initial positions of ions were randomized to the whole trap volume, reported trapping efficiencies would be significantly different, as trapping efficiency drops off with increased distance from the trap center. It is important to note, therefore, that trapping efficiencies reported herein are dependent on our choice of ionization volume. While these values are internally consistent within this paper, comparison with trapping efficiencies reported elsewhere require normalization with respect to the trapping volume and the ionization volume. Initial ion velocities and directions were randomized to a Maxwell distribution with a specified temperature. Ion time-of-birth was randomized with respect to the phase of the applied rf.

For simulations involving a collision gas, each gas molecule was defined to have random initial direction and velocity (also following a Maxwell distribution) at the moment of impact with an ion. The temperature of the collision gas was independently variable from the initial ion temperature; however, in all simulations both ions and neutrals started at the same temperature, as would be expected for direct (nondissociative) in situ ionization. Ions created via a dissociative process may have significantly greater initial kinetic energy and would likely not be trapped at all due to the shallow pseudopotential well. For simplicity, ions and neutrals were assumed to have diameters of 8 and $3 \AA$, respectively, corresponding approximately to the hard sphere diameters of toluene and nitrogen.

The collision frequency of ions with neutrals is given by the following relation:

$$
P=1-\exp \left(\frac{-\pi d^{2}\left(v_{i}+\bar{v}_{n}\right) \Delta t n}{\sqrt{2}}\right)
$$

which is derived in the Appendix. Ion-neutral collisions were assumed to be elastic. Scattering angles and velocities for each ion collision were calculated using exact three-dimensional momentum and energy conservation calculations. As momentum and energy provide only four constraints on the six unknown velocity components (three for the ion, three for the neutral), the remaining two components were chosen to be random- ized values of orthogonal scattering angles. The probability distributions of these randomized angles were derived from the two-dimensional projected area of impact parameter space, assuming hard spheres. This exact mechanical treatment is more accurate than simple viscous drag models [37], in which ion energy is reduced by a factor upon each collision, but the velocity vector does not change direction. Although a viscous drag model is adequate for situations in which ions are much faster than neutrals, for in situ ion formation, ion-neutral collisions result in changes in the direction of ion motion. The method used in the present work has been validated against, and shows agreement with the hard sphere collision model used by ITSIM (Ion Trap SIMulation) [19]. Details about the collision calculations are contained in the Appendix.

In most simulations, space-charge effects were not calculated. This represents the situation of an array of a large number of traps with a single ion created randomly in each trap. If ions are lost more quickly than they are formed in the traps, such will be the case, and ion-ion interactions can be ignored. In the simulations reported in section 3.3, several ions occupied the same trap simultaneously, and Coulombic forces between ions were calculated at each time step using the standard SIMION factor repulsion (with the factor set to 1). This setting most accurately models repulsion between any number of ions and is particularly applicable to the small volumes involved in these simulations. The simulations with space-charge included represent the situation in which ions are formed more quickly than they are lost, and a given trap frequently contains more than one ion at a time.

Computation levels in SIMION range from -500 to +500. A value of zero is the least accurate. Positive values include algorithms for field curvature detection, boundary checking, and velocity reversal detection, with +500 having the smallest computational (time) increment. Negative values do not include the aforementioned algorithms, but computational increments are similar, with -500 being the most accurate. In the present study simulations spanning the entire range of computational quality levels were studied to find an appropriate level to minimize computational time while maintaining accuracy. Simulations run at higher computational qualities (e.g., +150 and +500 ) showed $\sim 5 \%$ fewer ions trapped after $2000 \mathrm{rf}$ cycles than simulations run at low levels of computational quality $(0,-3$, and $-5)$. The additional ions lost at higher computational quality were usually lost during the first $100 \mathrm{rf}$ cycles of the trap, and loss rates beyond that point were identical between different computational levels. To minimize computation time, the majority of simulations reported herein were run at a level of -5 .

Except where noted, simulations were run for $2000 \mathrm{rf}$ cycles to calculate trapping efficiency. Ions were considered to be trapped if they remained in the trap for this length of time. However, at $\mathrm{GHz}$ frequencies, 2000 cycles represent a trapping time about a microsecond. 
To examine the ability of micro-traps to store ions for time scales about a millisecond, a few simulations were run for $10^{6}$ to $10^{7} \mathrm{rf}$ cycles. Trapping trends observed on the short time scale were similar to those observed at long time scales, although the absolute values of trapping efficiencies were different.

\section{Results and Discussion}

First we examine the behavior of isolated ions in a collisionless trapping environment, and later turn to the effects of pressure and Coulombic repulsion. Finally we present the effects of geometry defects. Each point in the following plots is the result of creating and attempting to trap 500 independent ions unless otherwise indicated. Error bars in the following plots represent \pm 1 standard deviation calculated using Poisson statistics. Trap voltages are reported as zero to peak $\left(\mathrm{V}_{0-p}\right)$, and $\mathrm{rf}$ frequencies are given in GHz.

\section{Ion Trapping in Vacuum}

Figure 3 shows regions of collisionless ion trapping as a function of trap voltage ( $\mathrm{rf}$ amplitude) and frequency. Ions were defined with $\mathrm{m} / \mathrm{z}=93 \mathrm{Th}$ and initial kinetic energies corresponding to $300 \mathrm{~K}$. Also shown, for reference, is the curve defined by $\mathrm{q}_{\mathrm{z}}=0.908$ (the Mathieu instability limit). Shaded regions indicate combinations of voltage and frequency for which trapping efficiency exceeds the indicated level. For electrical reasons it is desirable to operate the array of micrometer-sized ion traps at the lowest possible frequency and voltage, while keeping ion trapping at an acceptable level. When rf amplitude drops too low, the pseudopotential well is too shallow to trap ions, and as the rf amplitude and well depth increase, the probability that a given ion will be trapped increases.

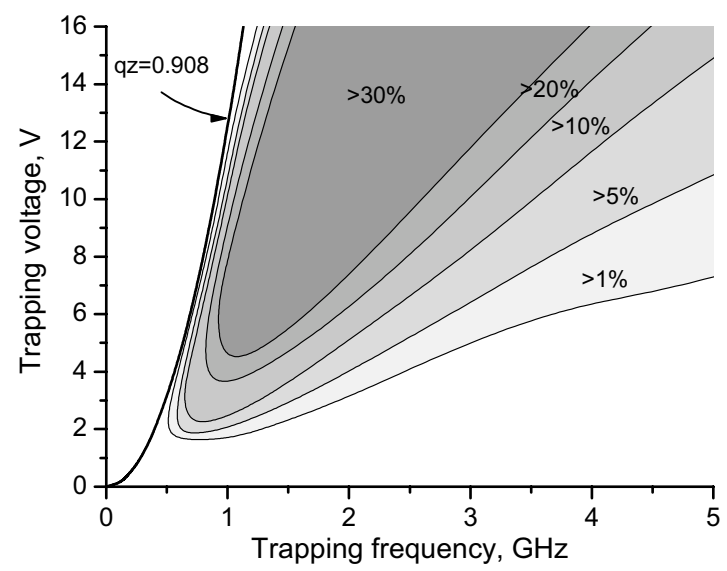

Figure 3. Calculated collisionless ion trapping efficiency as a function of $\mathrm{rf}$ amplitude (voltage) and operating frequency of a microfabricated cylindrical ion trap. Shaded regions indicate combinations of voltage and frequency for which trapping efficiency exceeds the indicated values. For reference, the parabola of constant $\mathrm{q}_{\mathrm{z}}$ is shown for $\mathrm{q}_{\mathrm{z}}=0.908$ (the Mathieu instability boundary).

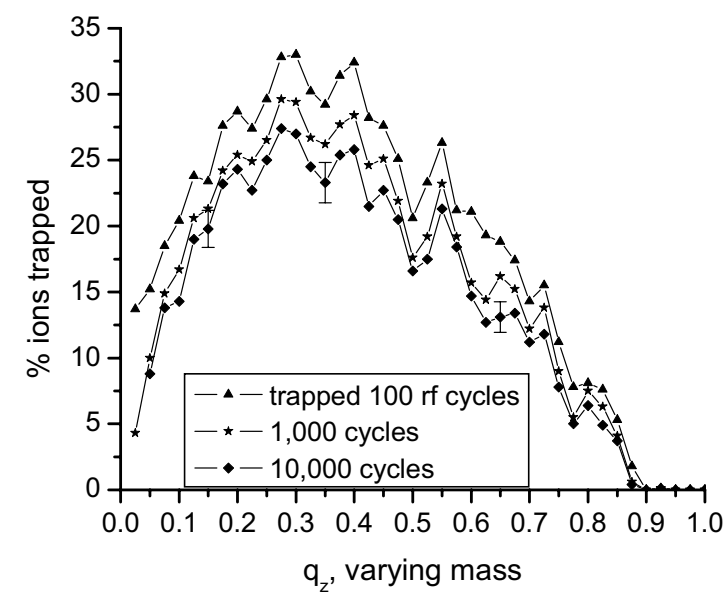

Figure 4. Calculated collisionless ion trapping efficiency as a function of the stability parameter, $\mathrm{q}_{\mathrm{z}}$. Ion mass is varied, while trap voltage and frequency are held constant at $8 \mathrm{~V}, 1.2 \mathrm{GHz}$, respectively. The same ions are followed through $10^{2}, 10^{3}$, and $10^{4}$ rf cycles. Each point represents the trapping efficiency of 500 ions.

Figure 4 show the collisionless ion trapping efficiency as a function of the stability parameter, $\mathrm{q}_{\mathrm{z}}$, in the absence of any DC offset $\left(a_{z}=0\right)$. Trap voltage and frequency were held constant at $1.2 \mathrm{GHz}$ and $8 \mathrm{~V}$, and the $\mathrm{m} / \mathrm{z}$ of ions was varied $(38-1500)$ to produce the indicated $\mathrm{q}_{\mathrm{z}}$ values. Varying trap parameters while ion mass was held constant produced a similar result. Ion trapping shows strong dependence on $\mathrm{q}_{\mathrm{z}}$, with a maximum at intermediate $\mathrm{q}_{\mathrm{z}}$ values and minima for both high and low values. This result corresponds qualitatively with escape velocity curves derived by Abraham and coworkers for ions in macroscopic traps [38]. At intermediate $\mathrm{q}_{\mathrm{z}}$ values the barrier for escape is higher, while in other regions the initial kinetic energy of ions, coupled with their phase and position, make escape more likely. Thus, for a given set of conditions in a micrometer-sized trap, trapping efficiency is somewhat mass selective. Note that values presented for the stability parameter, $\mathrm{q}_{\mathrm{z}}$, were calculated using the formula

$$
\mathrm{q}_{\mathrm{z}}=\frac{8 e V}{m\left(r_{0}^{2}+2 z_{0}^{2}\right) \Omega^{2}}
$$

which is only strictly true for an ideal hyperbolic trap. Thus, these $\mathrm{q}_{\mathrm{z}}$ values are only approximate.

Several simulations were carried through 1-2 million rf cycles. Ions continue to be lost for the entire trapping time, but loss rates steadily decline, such that ions can remain trapped for longer than $1 \mathrm{~ms}$ in a collisionless vacuum. Simulations of a few ions for 100 million rf cycles show some ions trapped for $100 \mathrm{~ms}$ in "perfect vacuum". Long-term trapping may not have application to portable mass spectrometry, where pressure effects will limit trapping time to values much shorter than this, but may have application to experiments in quantum computing and spectroscopy, where operat- 
ing pressures will be much lower. Presumably, as rf frequency increases (in this case, by 3 orders of magnitude), other processes will also be carried out on shorter time scales. For instance, trapping, cooling, and mass analysis should be faster. In this case, trapping times much less than $100 \mathrm{~ms}$ may be enough for trapping and analysis.

It is interesting to note that simulated ion trajectories precess about the z-axis with a non-constant rate. The magnitude of precession varies sinusoidally with a period of several hundred to a few thousand rf cycles, depending on the ion. This oscillation is not possible in a perfectly cylindrically-symmetric system. The noncylindrically-symmetric force acting on the ion motion appears to be the result of the mechanical and electrical connectors leading up to the ring electrodes, similar to the observation of oriented Wigner-type crystals in cold trapped ions [39]. It is not expected that this oscillation will have a significant effect on the ability of microfabricated traps to store, analyze, or detect ions.

\section{Ion Trapping with Collision Gas}

To understand the effect of ion-neutral collisions in a micrometer-sized ion trap, simulations examined ion trapping in the presence of helium, air, and other gases at pressures ranging from $1 \mathrm{mTorr}$ to several torr. Because of the small length scale of ion motion, collisions in micrometer-sized traps are extremely infrequent at pressures of a few mTorr, and many ions survive 2000 cycles having had no collisions at this pressure. Figure 5 shows ion trapping efficiency as a function of pressure for several trap voltage and temperature conditions. The collision gas in this case is air. The data for trapping efficiency as a function of pressure are fit well by limited growth curves of the form

$$
y=a \exp \left(-\exp \left(k \ln \left(P / P_{0}\right)\right)\right)
$$

where y represents the percentage of ions trapped, $a$ is the trapping efficiency at zero pressure, $P_{0}$ is the pressure at which trapping is reduced by $1 / e$, and $k$ is a fit parameter. These best-fit curves are included in the figure. In each case, trapping efficiency is reduced as pressure increases, and the collisionless environment represents the longest trapping time. Similarly, trapping efficiency is reduced as the mass of the neutral species (collision gas) increases. While a small pressure of cooling gas improves performance in larger ion trap systems where ions are injected into the trap, collisions reduce trapping efficiency and trapped ion lifetime for ions generated in situ in a micrometer-sized trap. Ironically, the pressure at which ion-neutral collisions begins to effect trapping efficiencies is on the same order as pressures used by macroscopic traps for optimal performance. In a macroscopic ion trap, collisions effectively cool trapped ions, and ions become concentrated at the center of the trap where nonlinear field components are minimized. In contrast, ions generated in situ in a microscopic ion trap are not cooled by the collisions they experience, and their trajectories remain farther from the center than they would be if cooled. Never-
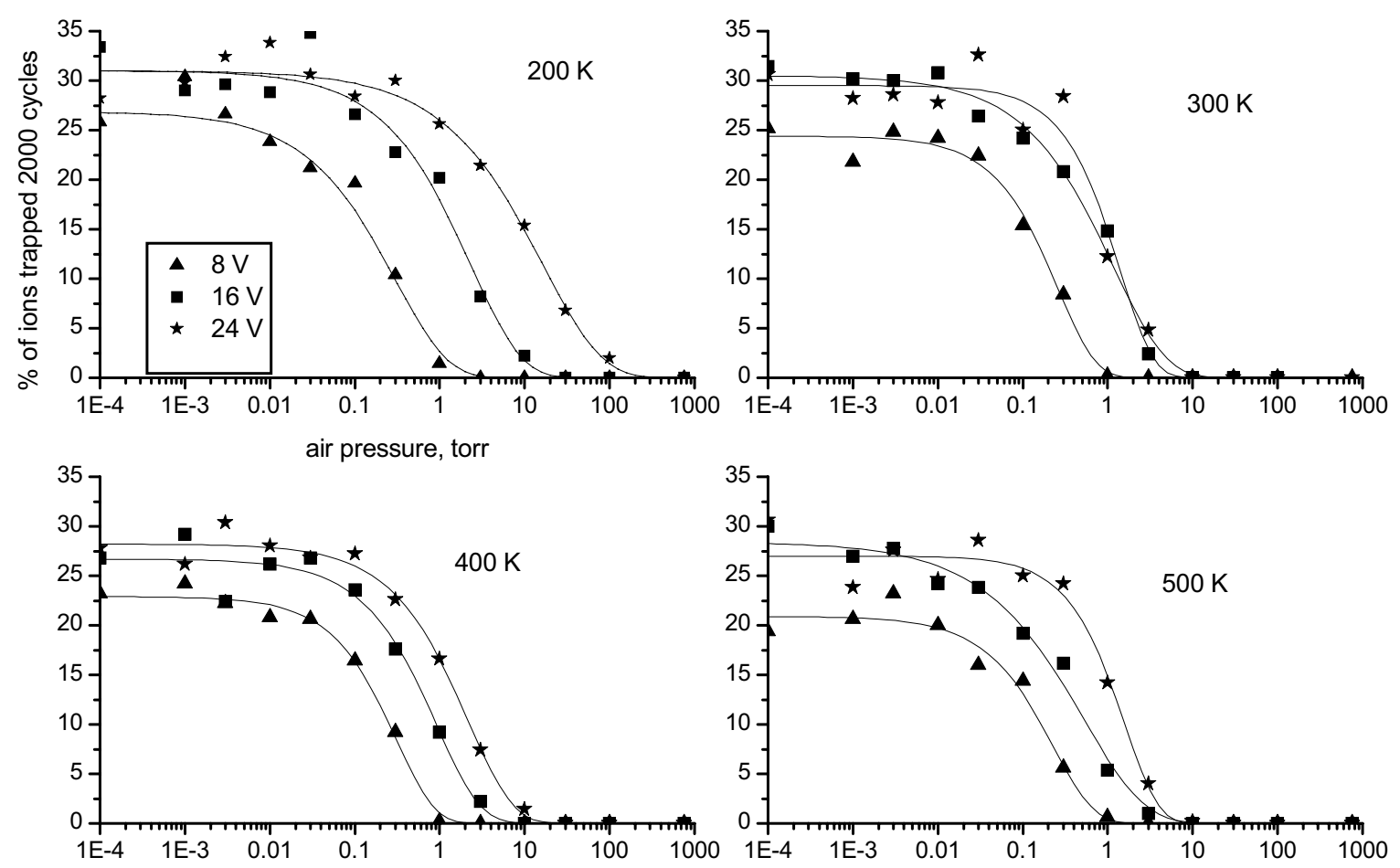

Figure 5. Calculated ion trapping efficiency as a function of pressure for several trap voltage and temperature conditions. Ions have $m / z=93$, neutral gas is air. Each point represents 500 ions. 
theless, depending on the length of time needed for ion storage and mass analysis, operation at pressures up to 1 torr may be possible in the micrometer-scaled trap. For instance, if $2000 \mathrm{rf}$ cycles are a sufficient amount of time for trapping and analysis, ion losses at 1 torr would be tolerable. If, however, longer times are needed for acceptable performance, such pressures may be detrimental.

The shallow pseudopotential well in a micrometersized trap (typically $0.3 \mathrm{~V}$ ) implies that thermal energies of ions may have a significant effect on ion trapping [19]. Simulations of ion trapping in which both the initial ion energies and collision gas temperature ( 1 torr helium) vary together from 200 to $500 \mathrm{~K}$ show this effect. As the initial kinetic energy of ions increases, trapping efficiency decreases. Additionally, an increased trap voltage results in improved ion trapping. Temperature may be an important consideration since any electrical heating of the trap would increase the neutral (and hence the ion) temperature. The effect of temperature on trapping illustrates another important issue, viz. that energetic ions resulting from dissociative ionization processes are likely not to be trapped under the conditions considered here.

\section{Space-Charge Effects}

The maximum density of singly-charged particles that can occupy an ideal hyperbolic ion trap is estimated by the relation [40]:

$$
N_{\max }=\frac{3 \varepsilon_{0} V_{0}^{2}}{4 m \Omega^{2} z_{0}^{4}}
$$

Whereas a macroscopic trap at modest trapping voltages can typically store $10^{3}-10^{6}$ ions, an ideal micrometer-sized trap has a calculated storage capacity of a few hundreds of ions. Simulations show, however, that only a single ion can be trapped for a useful length of time in a micrometer-sized cylindrical ion trap operated in the range of 5-20 V. Regardless of the number of ions created, after about $1 \mu \mathrm{s}$, only one ion remains trapped. For multiple ions in a given trap, each ion experiences Coulombic repulsion which eventually leads to an unstable trajectory and loss of all but one ion. The remaining ion no longer experiences any space-charge forces or effects. Simulations with ideal hyperbolic trap geometries, as well as traps with larger sizes $(2,5$, and $10 \mu \mathrm{m})$ show that this effect is general, but that the time scale of ion loss due to this mechanism increases rapidly as the trap size, rf amplitude, and field ideality increase. For instance, the average time required to lose all but one ion is $\sim 10 \mu \mathrm{s}$ for an ideal hyperbolic trap with $\mathrm{r}_{0}=1 \mu \mathrm{m}$, operated at $16 \mathrm{~V}$, and about $1 \mathrm{~ms}$ for a $10 \mu \mathrm{m}$ cylindrical trap at the same voltage. Thus the estimated charge density relation, $N_{\max }$ is true only for a relatively short time scale. For macroscopic traps this loss process also occurs, but slowly compared to a typical mass analysis experiment.

Ejection of all but one ion due to space charge has an interesting result: the single ion remaining in a trap tends to have a favorable combination of kinetic energy and trajectory such that it will likely be trapped for a long period of time in a collisionless environment. In addition, the remaining ion is no longer affected by space-charge from neighboring ions. To explore this phenomenon 100 ions were introduced into a trap during the first $\mathrm{rf}$ cycle, and Coulombic repulsion was calculated between each ion. Due to charge repulsion the trapped ion population was quickly reduced to a single, cooler ion. This simulation was repeated 100 times. After 10,000 rf cycles $(8.3 \mu \mathrm{s})$, in 97 of the 100 cases the trap still had a single trapped ion. Of those ions that remained after 10,000 cycles, $96 \%$ continued to be trapped for an additional 100,000 cycles. Although this simulation demonstrates the charge-induced ion loss process, it represents a physically improbable situation since ions cannot be formed within a trap at this rate.

A more realistic situation was simulated in which 25 argon ions were created at random times over defined periods ranging from $250 \mathrm{~ns}$ to $2.5 \mathrm{~ms}$, corresponding to average ionization rates of 100 to 0.1 ions per $\mu \mathrm{s}$. The trap was simulated with 5 mTorr argon, $8 \mathrm{~V}, 1.75 \mathrm{GHz}$. For each simulation, space-charge eliminated all but one ion, and the lifetime of the remaining ion was recorded. The simulation was repeated 20-100 times for each ionization rate. These results are shown in Figure 6 . The median lifetime of the remaining ion is equivalent to the time at which the trap has a $50 \%$ probability of being empty, and a 50\% probability of having a single ion. In no case did the trap have more than one ion at this point in time. When ionization rates are sufficiently fast, Coulombic repulsion selects the more favorably trapped ion, and rejects the others, similar to the evaporative cooling effect illustrated above. However, when the rate of ion formation is lower than the rate of ion loss, no net accumulation or selection of ions occurs in the traps. Consequently, successful operation of micrometer-sized traps will require ion formation rates exceeding ion loss rates. Of course, even one ion per microsecond per trap is a difficult level of in situ ionization to achieve. Because temperature, pressure, $\mathrm{q}_{\mathrm{z}}$, voltage, and other parameters affect ion lifetimes, they are also relevant in determining the necessary ion formation rate. That ion losses occur both including and ignoring space charge indicates that while space-charge may enhance this evaporative cooling effect, it is not necessary for this effect to exist (of course, space charge can be simply "turned off" in simulations, but not in the laboratory). Importantly, space-charge does not cool the remaining ion; it simply allows the selection of one favorably-trapped ion and rejection of all the other ions. For one randomly generated ion per trap, an array of traps will have a few traps with one trapped ion, while most traps will be empty. The remaining ions will have 


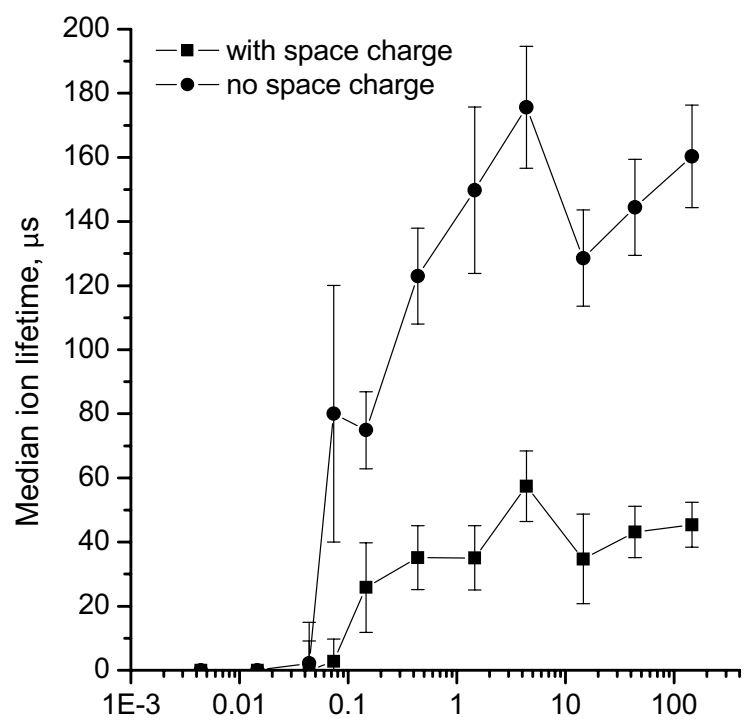

Average rate of ion formation, ions per $\mu \mathrm{s}$

Figure 6. Median ion lifetime as a function of the average ion formation rate. Argon ions in 5 mTorr argon, 1-micron trap simulated at $8 \mathrm{~V}, 1.75 \mathrm{GHz}$. Lower curve shows ions with space charge effects; in the upper curve space charge calculations were turned off. Ion lifetime begins at the end of the ionization event rather than at the moment the last ion is formed. Thus, for low ionization rates $(<0.1 \mathrm{ion} / \mu \mathrm{s})$, the median ion is already lost before the end of the ionization event, resulting in a lifetime of zero. Each point represents 500 ions. Error bars show 1 standard deviation calculated using Poisson statistics.

favorable trapping parameters (kinetic energy, position, etc.). Most traps, though, will be empty. On the other hand, if the same traps are each populated with several ions, after some time a majority of the traps will contain one trapped ion. Another result of charge-induced selection is that loss rates are more rapid immediately following the ionization event, and fewer stray ions will interfere with mass analysis and detection.

\section{Geometry Effects}

The geometry of these microfabricated traps was optimized using methods described earlier [19]. Geometry control in microfabrication has limitations not found in standard machining. Wall tapering, misalignment of layers, and limitations on the number and thickness of layers all have an effect on the trap geometry. The effects that such deviations might have on ion trapping were simulated using the methods described earlier in this paper, but with appropriately modified electrode arrays.

Fabrication of micrometer-sized traps using plasma etching produces an 85 to 87 degree sidewall taper on all trap electrodes. The direction of tapering is such that an electrode is wider at the top and narrower near the substrate. Although the taper results in an additional nonlinearity of the electric field in such a trap, simulations indicate that trapping efficiency is only minimally affected. Traps with a taper of 85 degrees were simu- lated under conditions of various voltage, frequency, and pressure conditions. The trajectories of ions showed visible distortion in tapered traps. However, the percent of ions trapped using tapered electrodes was generally only $1-3 \%$ lower than that for non-tapered electrodes under identical conditions, well within the error limits for each simulation. These results imply that the tapering of the electrode surfaces will have a negligible effect on ion trapping.

In cylindrical ion traps, the ratio of $z_{0}$ to $r_{0}$ can be varied over some range without significant modification of ion trapping properties [41]. This ratio was varied in simulations of micrometer-sized cylindrical ion traps operated at $8 \mathrm{~V}, 1.25 \mathrm{GHz}$. The value of $\mathrm{r}_{0}$ was held constant at $1 \mu \mathrm{m}$, and $\mathrm{z}_{0}$ was varied from 0.6 to 1.3 $\mu \mathrm{m}$. Under identical starting conditions ions were trapped for 25,000 rf cycles with no collision gas. Ion trapping shows a broad maximum for values of $z_{0}$ in the range 0.7 to $1.0 \mu \mathrm{m}$, and drops off gradually for values outside this range. Within limits of microfabrication, ion traps can in principle be constructed in a range of sizes, from $\mathrm{r}_{0}=1 \mu \mathrm{m}$ up to several micrometers, with $z_{0}$ scaling accordingly. It is suggested that ion trapping in larger traps will increasingly resemble trapping in macroscopic traps. However, factors such as electrical heating, structural mechanics, and operating pressure range vary differently and must be considered in designing larger microfabricated traps.

The misalignment of end cap electrodes with respect to the ring electrode is dictated by the alignment tolerance of the photolithography tool that defines the patterns. For the exposure tool used in this work (a GCA XLS stepper), the alignment tolerance is $150 \mathrm{~nm}$ $(3 \sigma)$ layer to layer, and is such that the maximum ring electrode to end cap misalignment would be $300 \mathrm{~nm}$. The results from the first microfabrication indicate that the actual misalignment (end cap to ring) is actually much smaller-about $50 \mathrm{~nm}$. Table 1 shows simulated ion trapping efficiencies for geometries involving layer misalignment. Trapping efficiencies are expressed as a fraction of the trapping in a perfectly-aligned trap. Data are shown for different misalignment modes with offsets of 50,100, and $150 \mathrm{~nm}$. Ion trapping is not noticeably reduced for $50 \mathrm{~nm}$ distortions, but begins to

Table 1. Normalized trapping efficiencies for electrode misalignments, expressed as a fraction of the trapping efficiency when no misalignment is present. Trapping efficiency is $25.4 \%$ with no misalignment. Error for each value is $+/-5 \%$

\begin{tabular}{lcccc}
\hline & \multicolumn{4}{c}{ Electrode displacement } \\
\cline { 2 - 5 } Nature of misalignment & $0 \mathrm{~nm}$ & $50 \mathrm{~nm}$ & $100 \mathrm{~nm}$ & $150 \mathrm{~nm}$ \\
\hline \hline Top endcap offset & 1.0 & 0.98 & 0.84 & 0.87 \\
Ring electrode offset & 1.0 & 0.98 & 0.75 & 0.61 \\
Bottom endcap offset & 1.0 & 0.98 & 0.88 & 0.74 \\
$\begin{array}{l}\text { Top and bottom offset in } \\
\text { opposite directions }\end{array}$ & 1.0 & 0.96 & 0.94 & 0.90 \\
$\begin{array}{l}\text { Top and bottom offset in } \\
\text { orthogonal directions }\end{array}$ & 1.0 & 1.0 & 0.89 & 0.83 \\
\hline
\end{tabular}


deteriorate for larger misalignments. These results imply that expected and actual process-induced misalignment of the end caps has a relatively minor effect on trapping efficiency for micrometer-scaled ion traps. Preliminary simulations of the effects of misalignment on ion ejection efficiencies also indicate a minor effect.

\section{Conclusions}

These simulations demonstrate that ion trapping is possible for micrometer-sized cylindrical ion traps. However, several aspects of micrometer-sized traps make them less effective than large traps for trapping ions. Shallow pseudopotential wells, necessitated by low voltage and power requirements, limit ionization to in situ methods. The shallow well coupled with spacecharge also limits ion storage capacity to a single ion per trap on the time scale needed for mass analysis and detection. Although a single trap is capable of trapping only one ion on a useful time scale, sensitivity is achieved by creating a large parallel array of traps. An array of $10^{6}$ traps with $\mathrm{r}_{0}=1 \mu \mathrm{m}$ has the same total trap volume as, but greater ion capacity than a single trap with $r_{0}=0.1 \mathrm{~mm}$. The standard mass-selectiveinstability scanning mode may be unable to achieve high mass resolution due to the absence of a sharp instability boundary at $\mathrm{q}_{\mathrm{z}}=0.908$. This gradual instability boundary also results in some mass selectivity in ion trapping and storage. As a consequence, good performance may require alternate mass analysis modes, such as resonance ejection or mass-selective trapping followed by pulse-out. Additionally, the difficulty of creating ions inside the small trap volume may require novel ionization methods.

Although nonlinear fields are present in all traps, particularly in cylindrical traps, the magnitude of the nonlinear contribution is zero at the trap center. Thus, ions that are cooled to the center of the trap experience minimal nonlinear effects. In micrometer-sized traps, however, thermal energies of ions result in a large ion excursion relative to the total trap volume. Ions spend significant time far from the trap center, and nonlinear effects may be great. Collisional cooling does not benefit trapping of ions generated in situ; however, tolerable pressures may be higher, due to the short path length and time scales involved than in conventional ion trap instruments, and pumping requirements may be reduced. Additionally, the issues of ionization rate, mass analysis mode, and signal detection sensitivity must be investigated before a working instrument is possible.

Likely, a micrometer is simply too small to make an effective trap. However, given current microfabrication technology, it is simpler to fabricate devices with dimensions typical of other MEMS devices, such as these micrometer-scale traps, than it is to produce traps in the size range of several to several tens of micrometers. Novel fabrication techniques may overcome this obstacle and allow development of larger traps. Micrometersize traps represent an extreme case, and obviously as traps can be made larger than this; their performance will be closer to the performance of macroscopic traps. Another obstacle apparent from this work is that the pseudopotential well depth cannot be arbitrarily small. While ion trapping is improved with higher operating voltages, microfabricated devices quickly reach upper voltage limits. On the other hand, the power required to drive those voltages can be reduced. It remains to be seen whether the advantages of ion traps of this size outweigh the disadvantages, including rapid ion loses, the requirement of in situ ionization, and the difficulty of ion formation and detection.

\section{Acknowledgments}

This research was funded by a Laboratory Directed Research and Development (LDRD) grant from Sandia National Laboratories. Sandia is a multiprogram laboratory operated by Sandia Corporation, a Lockheed Martin Company, for the United States Department of Energy's National Nuclear Security Administration under contract DE-AC04-94AL85000. The authors thank Sherry Zmuda for SEM analysis, and James G. Fleming and Melanie Tuck for device fabrication. The authors also thank Professor R. Graham Cooks and Guangxiang $\mathrm{Wu}$ of Purdue University for useful discussions.

\section{Appendix}

SIMION 7.0 [35] supports user-written programs that allow programmatic access to and control over most properties of ions and fields, as well as timing and computational parameters. In this way, time-varying electric fields, ion-neutral collisions, and randomization of initial ion properties are possible. This appendix outlines the specific algorithms used for the work presented here in specifying initial ion kinetic energies and positions, determining the outcome of ion-neutral collisions, and determining the number of collisions ions undergo. Note that SIMION treats all positions, velocities, etc. in Cartesian coordinates, so results in polar notation are converted to Cartesian in the program. In the following, the z-axis is the axis of (approximate) cylindrical symmetry of the trap.

\section{Initial Ion Velocities}

In determining initial ion velocities we assume that the ionization process does not change the kinetic energy or trajectory of the species being ionized, and we further assume that it was in thermal equilibrium before, and immediately upon ionization. The velocity distribution of ions under such conditions is a Maxwell, or spherical normal distribution, in which components in $(x, y, z)$ represent independent normal distributions with common variance and mean zero. Thus the Cartesian velocity components are represented by three independent Gaussian distribution functions of the form 


$$
f\left(v_{u}\right)=a e^{-b v_{u}^{2}}
$$

where $u$ designates $x, y$, or $z$; where $b$ is half the inverse of the variance, and has a value

$$
b=\frac{m}{2 k_{b} T^{\prime}}
$$

and where normalization of the distribution function yields $a$ with value

$$
a=\sqrt{\frac{b}{\pi}}=\sqrt{\frac{m}{2 \pi k_{b} T}}
$$

Implementing these results into a computational algorithm involves randomly generating values for $v_{x}$, $v_{y}$, and $v_{z}$ for each ion in such a way that the probability distribution function for each velocity component is Gaussian with the calculated variance. Standard computer random number generators, including that used in SIMION, produce random numbers $(X)$ with a flat probability distribution, and a range of $0-1$, thus the normalized distribution function is $f(x)=1$. The mean of $X$ is $\mu=1 / 2$. The variance, $\sim^{2}$, is defined as the value of the second central moment, and is calculated using

$$
\sigma^{2} \equiv E\left[(X-\mu)^{2}\right]=\int_{0}^{1} x^{2}(f(x)-\mu)^{2} d x=\frac{1}{4} \int_{0}^{1} x^{2} d x=\frac{1}{12} .
$$

According to the Central Limit Theorem, the sum $\left(Y_{n}\right)$ of a large number $(n)$ of such random numbers has an approximately Gaussian probability distribution centered at $n / 2$. Because variances of uncorrelated random variables are additive, the variance of $Y_{n}$ is $n / 12$. Visual inspection of such distributions shows that $n=5$ suffices for the purposes of this program. We define the scaling function $g\left(Y_{n}\right)=c Y_{n}$ where $c$ is used to set the variance of $g\left(Y_{n}\right)$ equal to the variance of the desired velocity distributions of ions. The value of $c$ is determined by:

$$
\frac{k_{b} T}{m}=\frac{n}{12} c
$$

and finally the velocity component is calculated using the relation

$$
v_{u}=\left(Y_{n}-\frac{n}{2}\right) \sqrt{c}
$$

\section{Initial Ion Positions}

Ions originate within a cylindrical volume of radius $R$ and length $L$, which are entered at the start of each simulation. For each ion, the radial position, $r$, is deter- mined using the square root of a single random number, scaled by the radius of the desired cylinder, $r=$ $R \sqrt{X}$. The ion is then assigned a random angle, $\phi$, with range $0-2 \pi$. The $x$ and $y$ coordinates of the ion are then determined using $r \sin \phi$ and $r \cos \phi$, respectively. The $z$ component of the ion is simply a random number scaled by $L$. The resulting distribution is equivalent to that made by taking a flat distribution within a rectangular solid and explicitly excluding those ions that fall outside of an inscribed cylinder, which is the method used by the program ITSIM (Guangxiang $\mathrm{Wu}$, personal communication, 2004).

\section{Collision Mechanics}

The collision frequency of ions with neutrals is a function of the mean relative velocity $\langle v\rangle$ between ions and neutrals [42]:

$$
z=\pi d^{2}\langle v\rangle n
$$

where $n$ represents the number density of neutrals, $d_{A}$ and $d_{B}$ represent the hard sphere diameters of the ion and neutral, and $d$ is the average diameter:

$$
d=\frac{1}{2}\left(d_{A}+d_{B}\right)
$$

The velocity of each ion at each time step, represented by the vector $v_{i}$, is known, although it changes rapidly in time with the applied $\mathrm{rf}$ and the relative phases and amplitudes of all aspects of the ion motion, such as the secular frequency and ion micromotion. The mean velocity of neutrals $\left(\left(\bar{v}_{n}\right)\right)$ is defined by the neutral temperature, which is input at the start of each simulation. The velocity distribution function for the neutrals is

$$
F\left(v_{n}\right) d v_{n}=4 \pi\left(\frac{m}{2 \pi k T}\right)^{\frac{3}{2}} \exp \left(\frac{-m v^{2}}{2 k T}\right) v_{n}^{2} d v_{n}
$$

for which the mean velocity is

$$
\bar{v}_{n}=\int_{0}^{\infty} v_{n} F\left(v_{n}\right) d v_{n}=\sqrt{\frac{8 R T}{\pi M}} .
$$

In the simulations this function is approximated as described above (eqs A-5 and A-6). The joint distribution function is simply the product of the ion velocity distribution function and the neutral velocity distribution. Of course, for a given ion and a given time step in the simulation, the distribution function is simply the delta function at the current value of the ion velocity, such that the joint distribution function is

$$
F\left(v_{i}, v_{n}\right)=\delta\left(v_{i}\right) F\left(v_{n}\right)
$$


The mean relative velocity to be used for calculation of collision frequency is therefore the integral of the product of the joint distribution function and the relative velocity of ions to neutrals, $v$ :

$$
\langle v\rangle=\int_{0-\infty} v F\left(v_{n}\right) \delta\left(v_{i}\right) d v_{n} d v_{i}
$$

which cannot be solved using the property

$$
\int_{0}^{\infty} f(x) \delta(x-a) d x=f(a)
$$

because the ion velocity and neutral velocity spaces are not equivalent. However, using the fact that the integral of the delta function is unity gives

$$
\begin{aligned}
& \langle v\rangle=\int_{0}^{\infty} v F\left(v_{n}\right) d v_{n} \\
& \langle v\rangle=4 \pi\left(\frac{m_{n}}{2 \pi k_{b} T_{n}}\right)^{\frac{3}{2}} \int_{0}^{\infty} v \exp \left[\frac{-m_{n} v_{n}^{2}}{2 k_{b} T_{n}}\right] v_{n}^{2} d v_{n} .
\end{aligned}
$$

The relative velocity of ions to neutrals is equal to the vector difference,

$$
v=\left|v_{i}-v_{n}\right|
$$

with the center of mass moving with velocity

$$
V=\frac{v_{i}+v_{n}}{2}
$$

We have an integral of the form

$$
\int_{0}^{\infty}(a-x) e^{-b x^{2}} x^{2} d x
$$

which must be solved numerically. We approximate the solution to eq A-12 with:

$$
\langle v\rangle=\frac{\sqrt{2}}{2}\left(v_{i}+\bar{v}_{n}\right)=\frac{v_{i}+\bar{v}_{n}}{\sqrt{2}} .
$$

In the limit that the ion is part of an ion distribution with velocities equal to those of the neutrals (either from the same temperature and the same mass, or both mass and temperature different but such that the velocities are the same-differences in size being taken into account from $\pi d^{2}$ ), that is, $\bar{v}_{i}=\bar{v}_{n}$ then

$$
\langle v\rangle=\frac{\sqrt{2}}{2}\left(\bar{v}_{n}+\bar{v}_{n}\right)=\sqrt{2} \bar{v}_{n}
$$

which is the standard result from kinetic theory [42]. In the present simulations the collision frequency per ion, $z$, is calculated using the following relation:
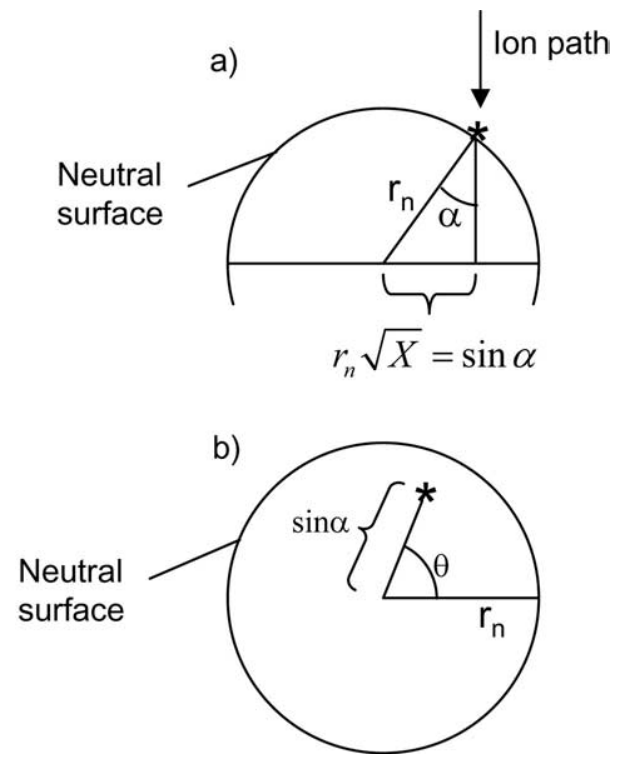

Figure 7. Geometry of ion-neutral impact showing (a) the plane containing the ion path and the center of the neutral, and (b) the neutral as seen by the approaching ion.

$$
z=\frac{\pi d^{2}\left(v_{i}+\bar{v}_{n}\right) n}{\sqrt{2}} .
$$

Thus the determination of whether a collision between an ion and a neutral occurred during any given time step $(\Delta t)$ was based on the probability function:

$$
P=1-\exp \left(\frac{-\pi d^{2}\left(v_{i}+\bar{v}_{n}\right) \Delta t n}{\sqrt{2}}\right) .
$$

Once the program determines that a collision takes, the program uses the following procedure to determine the velocity components of the ion after the collision. The velocity components of the neutral species before impact are determined using the same method as that used for initial ion properties. Following that, the ion trajectory (before collision) is put into the reference frame of the neutral species, with the orientations of the $x, y$, and $z$ axes remaining the same as the SIMION workbench. The neutral species (which is now at rest), is assumed to be a sphere with radius $r_{n}$. The ion approaches the neutral at an angle, $\beta$, which SIMION treats as a combination of elevational (el) and azimuthal (az) angles. At the moment of impact, momentum is transferred from the ion to the neutral in the direction normal to the surface of the sphere at the point of impact. Because the neutral is stationary before impact (in the frame of reference which we have thus defined), the direction with which momentum is transferred becomes the direction of motion for the neutral after the collision. Let this angle be called $\alpha$, defined as the angle between the pre-collision ion motion and the postcollision neutral motion. Figure 7 diagrams the collision from two angles: the top schematic (Figure 7a) shows 
the plane containing both the center of the neutral and the line containing the trajectory of the ion before collision, while the bottom schematic (Figure $7 \mathrm{~b}$ ) shows the neutral as seen from the approaching ion (the ion is out of the page). The point of impact is represented by an asterisk $\left(^{*}\right)$. The radial distribution of the impact point is $r_{n} \sqrt{X}$, while the angular distribution, $\theta$, is randomized through $2 \pi$ angles (determined using $\theta=$ $2 \pi X)$. Note that $\sin \alpha=r_{n} \sqrt{X}$. The elevational and azimuthal components of the neutral motion are determined using the relations:

$$
\begin{aligned}
& \mathrm{el}=\beta+\alpha \sin \theta \\
& \mathrm{az}=\beta+\alpha \cos \theta
\end{aligned}
$$

which angles are then converted into ratios $\mathrm{v}_{\mathrm{x}} / \mathrm{v}_{\mathrm{y}}$ and $\mathrm{v}_{\mathrm{y}} / \mathrm{v}_{\mathrm{z}}$ for the neutral. The reference for angle $\theta$ is arbitrary, but cancels out since $\theta$ spans $2 \pi$. Also, the orientation of the components $\sin \theta$ and $\cos \theta$ with respect to the SIMION coordinate system is arbitrary, but cancels out, so a rigorous transformation is not needed.

This calculation can be approached in a much simpler fashion, while still producing the same distribution of scattering angles and velocities. The probability distribution of the sine of a random number $(Y=$ $\sin [X])$ is identical to the distribution of forward/ backward scattering angles for ions after a collision. Thus, after conversion to the frame of reference of the neutral at rest, the forward scattering angle can be found by scaling the sine of a random number to the maximum scattering angle, $\theta_{\max }$, which is $\theta_{\max }=m_{\text {neutral }} /$ $m_{\text {ion }}$ if the ion is more massive than the neutral, and $\theta_{\max }$ $=\pi$ in all other cases. This alternate algorithm was realized after the present studies commenced, and so was not used.

For two-body elastic collisions, conservation of momentum and energy provide four constraints on the six variables (three velocity components for the ion, three for the neutral). The randomized values $v_{x} / v_{y}$ and $\mathrm{v}_{\mathrm{y}} / \mathrm{v}_{\mathrm{z}}$ of the neutral species provide the other two constraints. Solving these equations gives the following for the changes in the ion velocity components upon collision:

$$
\begin{aligned}
& \Delta v_{x_{i}}=\left(\frac{m_{n}}{m_{i}}\right)\left(\frac{-2\left(v_{x_{i}}+v_{z_{i}} U+v_{y_{i}} W\right)}{\left(\frac{m_{n}}{m_{i}}+1\right)\left(U^{2}+W^{2}+1\right)}\right) \\
& \Delta v_{y_{i}}=\left(\frac{W m_{n}}{m_{i}}\right)\left(\frac{-2\left(v_{x_{i}}+v_{z_{i}} U+v_{y_{i}} W\right)}{\left(\frac{m_{n}}{m_{i}}+1\right)\left(U^{2}+W^{2}+1\right)}\right) \\
& \Delta v_{z_{i}}=\left(\frac{U m_{n}}{m_{i}}\right)\left(\frac{-2\left(v_{x_{i}}+v_{z_{i}} U+v_{y_{i}} W\right)}{\left(\frac{m_{n}}{m_{i}}+1\right)\left(U^{2}+W^{2}+1\right)}\right)
\end{aligned}
$$

where $m_{n}$ and $m_{i}$ are the mass of neutral and ion, respectively, and $U$ and $W$ are the ratios of postcollision neutral velocities defined as:

$$
\begin{gathered}
U=\frac{v_{z_{n}}}{v_{x_{n}}} \\
W=\frac{v_{y_{n}}}{v_{x_{n}}} .
\end{gathered}
$$

Subsequently, the ion velocity components are restored to the lab frame of reference. In this computer program the ion color also changed upon each collision, allowing visual observations of collisions.

\section{Collision Counting}

SIMION is unable to export information other than the values of reserved variables (e.g., ion properties) at given points in time, so the collision count had to piggyback on a reserved variable. The number of collisions each ion experienced was tracked in the following manner: each time an ion collided with a neutral, the mass of the ion was incremented by $10^{-9}$ amu. At the end of each simulation the mass of each ion was recorded. The outcomes of simulations did not appear to be affected by this small increase in mass.

\section{References}

1. Cornish, T. J.; Ecelberger, S.; Brinckerhoff, W. Miniature Time-of-Flight Mass Spectrometer Using a Flexible Circuitboard Reflector. Rapid Commun. Mass Spectrom. 2000, 14, 2408-2411.

2. Austin, D. E.; Ahrens, T. J.; Beauchamp, J. L. Dustbuster: A Compact Impact-Ionization Time-of-Flight Mass Spectrometer for in Situ Analysis of Cosmic Dust. Rev. Sci. Instrum. 2002, 73(1), 185-189.

3. Austin, D. E.; Manning, H. L. K.; Bailey, C. L.; Beauchamp, J. L. A Compact Time-of-Flight Mass Spectrometer for High-Flux Cosmic Dust Analysis. J. Geophys. Res. Planets 2004, 109.

4. Bryden, W. A.; Benson, R. C.; Ecelberger, S. A.; Phillips, T. E.; Cotter, R. J.; Fenselau, C. The Tiny-TOF Mass Spectrometer for Chemical and Biological Sensing. Johns Hopkins APL Technical Digest 1995, 16(3), 296-310.

5. Cotter, R. J.; Fancher, C.; Cornish, T. J. Miniaturized Time-of-Flight Mass Spectrometer for Peptide and Oligonucleotide Analysis. J. Mass Spectrom. 1999, 34(12), 1368-1372.

6. Berkout, V. D.; Cotter, R. J.; Segers, D. P. Miniaturized EI/Q/oa TOF Mass Spectrometer. I. Am. Soc. Mass Spectrom. 2000, 12(6), 641-647.

7. Diaz, J. A.; Giese, C. F.; Gentry, W. R. Sub-Miniature ExB Sector-Field Mass Spectrometer. J. Am. Soc. Mass Spectrom. 2001, 12, 619-632.

8. Orient, O. J.; Chutjian, A.; Garkanian, V. Miniature High-Resolution Quadrupole Mass Spectrometer Array. Rev. Sci. Instrum. 1997, 68(3), 1393-1397.

9. Patterson, G. E.; Guymon, A. J.; Riter, L. S.; Everly, M.; Griep-Raming, J.; Laughlin, B. C.; Zheng, O. Y.; Cooks, R. G. Miniature Cylindrical Ion Trap Mass Spectrometer. Anal. Chem. 2002, 74(24), 6145-6153.

10. Badman, E. R.; Cooks, R. G. Cylindrical Ion Trap Array with Mass Selection by Variation in Trap Dimensions. Anal. Chem. 2000, 72(20), 5079-5086.

11. Badman, E. R.; Cooks, R. G. A Parallel Miniature Cylindrical Ion Trap Array. Anal. Chem. 2000, 72(14), 3291-3297.

12. Badman, E. R.; Johnson, R. C.; Plass, W. R.; Cooks, R. G. A Miniature Cylindrical Quadrupole Ion Trap: Simulation and Experiment. Anal. Chem. 1998, 70(23), 4896-4901.

13. Orient, O. J.; Chutjian, A. A Compact, High-Resolution Paul ion Trap Mass Spectrometer with Electron-Impact Ionization. Rev. Sci. Instrum. 2002, 73(5), 2157-2160.

14. Moxom, J.; Reilly, P. T. A.; Whitten, W. B.; Ramsey, J. M. Double Resonance Ejection in a Micro Iion Trap Mass Spectrometer. Rapid Commun. Mass Spectrom. 2002, 16(8), 755-760.

15. Kornienko, O.; Reilly, P. T. A.; Whitten, W. B.; Ramsey, J. M. Electron Impact Ionization in a Micro Ion Trap Mass Spectrometer. Rev. Sci. Instrum. 1999, 70(10), 3907-3909. 
16. Kornienko, O.; Reilly, P. T. A.; Whitten, W. B.; Ramsey, J. M. Micro Ion Trap Mass Spectrometry. Rapid Commun. Mass Spectrom. 1999, 13, 50-53

17. Moxom, J.; Verbeck, G. F.; Whittein, W. B. Miniaturization of Mass Spectrometers Based on Submillimeter Cylindrical Ion Traps. Proceedings of the 53rd ASMS Conference; San Antonio, TX, June 2005; Abstract AO51112.

18. Whitten, W. B.; Reilly, P. T. A.; Ramsey, J. M. High-Pressure Ion Trap Mass Spectrometry. Rapid Commun. Mass Spectrom. 2004, 18(15), 17491752.

19. Blain, M. G.; Riter, L. S.; Cruz, D.; Austin, D. E.; Wu, G.; Plass, W. R.; Cooks, R. G. Towards the Hand-Held Mass Spectrometer: Design Considerations, Simulation, and Fabrication of Micrometer-Scaled Cylindrical Ion Traps. Int. J. Mass Spectrom. 2004, 236(1), 91-104.

20. Cruz, D.; Blain, M. G. Field Emission Characteristics of a Tungsten Microelectromechanical System Device. Appl. Phys. Lett. 2005, 86(15), Art. no. 153502

21. Badman, E. R.; Cooks, R. G. Special Feature: Perspective-Miniature Mass Analyzers. J. Mass Spectrom. 2000, 35, 659-671.

22. Lee, W. W.; Oh, C. H.; Kim, P. S.; Yang, M.; Song, K. S. Characteristics of Cylindrical Ion Traps. Int. J. Mass Spectrom. 2003, 230(1), 25-31.

23. Yoshinari, K. Theoretical and Numerical Analysis of the Behavior of Ions Injected into a Quadrupole Ion Trap Mass Spectrometer. Rapid Commun. Mass Spectrom. 2000, 14(4), 215-223.

24. Baril, M.; Le, R.; Marchand, P. An Improved and Accurate Method for the Calculation of Trajectories in Quadrupole Mass Filters and Ion Traps Using Phase-Space Cynamics. Int. J. Mass Spectrom. Ion Processes 1990, 98(1), 87-97.

25. Reiser, H. P.; Julian, R. K.; Cooks, R. G. A Versatile Method of Simulation of the Operation of Ion Trap Mass Spectrometers. Int. J. Mass Spectrom. Ion Processes 1992, 121(1/2), 49-63.

26. Perrier, P.; Nguema, T.; Carette, M.; Andre, J.; Zerega, Y.; Brincourt, G.; Catella, R. New Operating Mode of a Qquadrupole Ion Trap in Mass Spectrometery. 4. Simulation Studies. Int. J. Mass Spectrom. 1997, 171(1/ 3), 19-29.

27. Ding, L.; Sudakov, M.; Kumashiro, S. A Simulation Study of the Digital Ion Trap Mass Spectrometer. Int. J. Mass Spectrom. 2002, 221(2), 117-138.

28. Sheretov, E. P.; Philippov, I. V.; Karnav, T. B.; Ivanov, V. W. Some Properties of Ion Trajectories in the Radial Plane of an Axially Symmetric Ion Trap. Rapid Commun. Mass Spectrom. 2002, 16(17), 1658-1667.

29. Nappi, M.; Weil, C.; Cleven, C. D.; Horn, L. A.; Wollnik, H.; Cooks, R. G. Visual Representations of Simulated Three-Dimensional Ion Trajecto- ries in an Ion Trap Mass Spectrometer. Int. J. Mass Spectrom. Ion Processes 1997, 161(1/3),77-85.

30. March, R. E.; Londry, F. A.; Alfred, R. L.; Franklin, A. M.; Todd, J. F. J. Mass-Selective Isolation of Ions Stored in a Quadrupole Ion Trap-a Simulation Study. Int. J. Mass Spectrom. Ion Processes 1992, 112(2/3), 247-271.

31. Londry, F. A.; Alfred, R. L.; March, R. E. Computer-Simulation of Single-Ion Trajectories in Paul-Type Ion Traps. J. Am. Soc. Mass Spectrom. 1993, 4(9), 687-705.

32. Lee, W. W.; Min, S.-K.; Oh, C. H.; Kim, P. S.; Song, S. H.; Yang, M.; Song, K. Stability of Ion Motion in the Quadrupole Ion Trap Driven by Rectangular Waveform Voltages. Int. J. Mass Spectrom. 2003, 230, 65-70.

33. Tabert, A. M.; Griep-Raming, J.; Guymon, A. J.; Cooks, R. G. HighThroughput Miniature Ccylindrical Ion Trap Array Mass Spectrometer. Anal. Chem. 2003, 75(21), 5656-5664.

34. Riter, L. S.; Peng, Y. A.; Noll, R. J.; Patterson, G. E.; Aggerholm, T.; Cooks, R. G. Analytical Performance of a Miniature Cylindrical Ion Trap Mass Spectrometer. Anal. Chem. 2002, 74(24), 6154-6162.

35. Moxom, J.; Reilly, P. T. A.; Whitten, W. B.; Ramsey, J. M. Analysis of Volatile Organic Compounds in Air with a Micro Ion Trap Mass Analyzer. Anal. Chem. 2003, 75(15), 3739-3743.

36. Dahl, D. A. version 6.0 and 7.0 eds.; Idaho National Engineering and Environmental Laboratory: Idaho Falls, ID, 2000.

37. Quarmby, S. T.; Yost, R. A. Fundamental Studies of Ion Injection and Trapping of Electrosprayed Ions on a Quadrupole Ion Trap. Int. J. Mass Spectrom. 1999, 190/191, 81-102.

38. Abraham, G. T.; Chatterjee, A.; Menon, A. G. Escape Velocity and Resonant Ion Dynamics in Paul Trap Mass Spectrometers. Int. J. Mass Spectrom. 2004, 231, 1-16.

39. Wineland, D. J.; Bergquist, J. C.; Itano, W. M.; Bollinger, J. J.; Manney, C. H. Atomic-Ion Coulomb Clusters in an Ion Trap. Phys. Rev. Lett. 1987, 59(26), 2935-2938.

40. March, R. E.; Hughes R. J. In Chemical Analysis: A Series of Monographs on Analytical Chemistry and Its Applications; Winefordner, J. D., Ed.; Wiley: New York, 1989; Vol. CII, p 305.

41. Fulford, J. E.; March, R.; Mather, R.; Todd, J.; Waldren, R. M. The Cylindrical Ion Trap. Can. J. Spectroscop. 1980, 25(4), 85.

42. Noggle, J. H. Physical Chemistry, 3rd ed.; Harper Collins: New York, 1996, pp 451-455. 